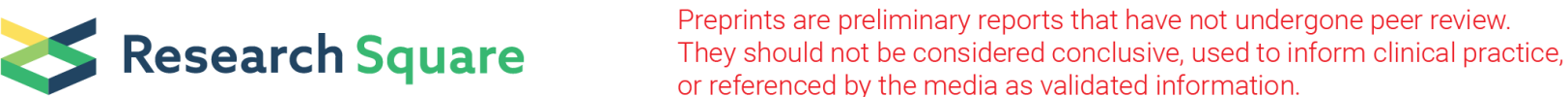

\section{Assessment of Therapeutic Outcome and Role of Reirradiation in Patients With Radiation-induced Glioma}

Makoto Ohno ( $D$ mohno@ncc.go.jp)

National Cancer Center Hospital: Kokuritsu Gan Kenkyu Center Chuo Byoin https://orcid.org/00000001-8031-4306

\section{Yasuji Miyakita}

National Cancer Center Hospital: Kokuritsu Gan Kenkyu Center Chuo Byoin

Masamichi Takahashi

National Cancer Center Hospital: Kokuritsu Gan Kenkyu Center Chuo Byoin

\section{Shunsuke Yanagisawa}

National Cancer Center Hospital: Kokuritsu Gan Kenkyu Center Chuo Byoin

\section{Yukie Tamura}

National Cancer Center Hospital: Kokuritsu Gan Kenkyu Center Chuo Byoin

Daisuke Kawauchi

National Cancer Center Hospital: Kokuritsu Gan Kenkyu Center Chuo Byoin

\section{Miyu Kikuchi}

National Cancer Center Hospital: Kokuritsu Gan Kenkyu Center Chuo Byoin Hiroshi Igaki

National Cancer Center Hospital: Kokuritsu Gan Kenkyu Center Chuo Byoin Akihiko Yoshida

National Cancer Center Hospital: Kokuritsu Gan Kenkyu Center Chuo Byoin Kaishi Satomi

National Cancer Center Hospital: Kokuritsu Gan Kenkyu Center Chuo Byoin Yuko Matsushita

National Cancer Center Hospital: Kokuritsu Gan Kenkyu Center Chuo Byoin Koichi Ichimura

National Cancer Center Hospital: Kokuritsu Gan Kenkyu Center Chuo Byoin Yoshitaka Narita

National Cancer Center Hospital: Kokuritsu Gan Kenkyu Center Chuo Byoin 
Keywords: radiation-induced glioma, IDH1/2 mutations, secondary neoplasms, long-term survivors of childhood malignancies, reirradiation

Posted Date: December 30th, 2021

DOI: https://doi.org/10.21203/rs.3.rs-1204208/v1

License: (1) This work is licensed under a Creative Commons Attribution 4.0 International License. Read Full License 


\section{Abstract}

Background: We retrospectively reviewed the clinical characteristics and treatment outcomes to clarify the optimal therapeutic strategy, especially the role of reirradiation in patients with radiation-induced glioma (RIG).

Methods: We identified patients with high-grade glioma who satisfied Cahan's criteria for RIG in our database during 2001-2021 and analyzed the outcomes.

Results: We identified 11 patients with RIG. The primary diseases included germinomas $(n=2)$, acute lymphoblastic lymphomas $(n=2)$, medulloblastomas $(n=3)$, diffuse astrocytoma with isocitrate dehydrogenase $(I D H) 2$ mutant $(n=1)$, pilocytic astrocytoma $(n=1)$, pituitary adenoma $(n=1)$, and a metastatic tumor from lung cancer $(n=1)$. The median latency period was 17 years (range: $9-30$ years). The RIGs included glioblastoma with IDH $1 / 2$ wild-type $(n=7)$, glioblastoma not otherwise specified $(n=2)$, anaplastic astrocytoma with $I D H 1 / 2$ wild-type $(n=1)$, and anaplastic astrocytoma not otherwise specified $(n=1)$. All patients underwent tumor removal or biopsy, 5 patients postoperatively received reirradiation combined with chemotherapy, and 6 patients were treated with chemotherapy alone. The median progression-free and overall survival times were 11.3 and 28.3 months, respectively. The median progression-free survival time of patients treated with reirradiation and chemotherapy $(n=5)$ tended to be longer than that of patients that received chemotherapy alone $(n=6)$ (17.0 vs 8.1 months; $p=0.45)$; the median survival time was similar (29.6 vs 27.4 months; $p=0.28$ ). Local recurrences were observed less frequently in patients who received reirradiation combined with chemotherapy (50\%) than in those who received chemotherapy alone $(100 \% ; p=0.046)$. None of the patients developed radiation necrosis. In one case, the different IDH2 mutational states between the primary and secondary tumors were useful for diagnosing the secondary tumor as RIG.

Conclusions: RIG can occur more than 20 years after successful treatment of the primary disease using radiotherapy; thus, follow-up times should be extended to 30 years. Reirradiation combined with chemotherapy appears to be feasible and have favorable outcomes. Identifying the IDH1/2 mutational status can have a diagnostic effect on establishing RIG in recurrent gliomas.

\section{Background}

Radiotherapy is used for cancer treatments, including pediatric brain tumors and hematological malignancies, such as glioma, medulloblastoma, germ cell tumors, and leukemia. Despite an overall improvement in the survival rates of patients with these tumors, patients treated with radiotherapy are at risk of long-term neurological complications such as the development of progressive leukoencephalopathy, arteritis, hypopituitarism, and hypothalamic insufficiency [1]. One of the most serious late consequences of radiotherapy is secondary neoplasms, which occur in rare cases but represents a major cause of mortality in long-term survivors of childhood malignancies [2-6]. Among radiation-induced brain tumors, meningiomas and gliomas are the most frequently reported secondary 
neoplasms [1]. The cumulative risk of secondary brain tumors occurring after radiation therapy for pituitary adenomas is $2.0 \%$ at 10 years and $2.4 \%$ at 20 years, which is 10.5 times higher than that seen in the general population [3]. The cumulative risk of secondary brain tumors occurring among long-term survivors of acute lymphoblastic leukemia is $0.8 \%$ at 10 years and $1.87 \%$ at 20 years [2].

Radiation-induced gliomas (RIGs) are typically high-grade tumors. The median latency period for developing RIGs is 9 years, and most RIGs occur within 15 years [7]. The treatments of RIGs are usually challenging, and the clinical outcomes are generally poor $[3,5,6,8]$. The median survival time (MST) of patients with RIGs is 11 months with a 2-year survival rate of $20.2 \%$ [6]. Several studies and review articles have proposed a combination therapy of reirradiation (ReRT) and chemotherapy as a potential treatment option; however, there are few reports on the detailed treatment outcomes of this combined therapy. Thus, the optimal therapeutic approach for RIGs is not well defined [6]. Moreover, there are few studies that investigated genetic alterations in RIGs [9-15], and their clinical impact remain unclear.

In this study, we retrospectively analyzed the clinical characteristics, treatment outcomes, and genetic alterations of 11 patients with RIG to clarify the optimal follow-up period for the treatment of the primary disease, optimal therapeutic strategy, especially for the role of ReRT, and the clinical impact of genetic characteristics in RIG.

\section{Methods And Materials}

\section{Patient characteristics}

This study was a retrospective observational study. We reviewed our departmental database between 2001 and 2021. We included patients who satisfied Cahan's criteria, which were as follows: (1) the tumor must originate in a previously irradiated region (but not necessarily in the full-dose region), (2) there must be a sufficient latency time between irradiation and the onset of the postradiation tumor, (3) the tumor histology must be different from that of the primary tumor, and (4) the patient must not have pathologies that favor the development of tumors: Li-Fraumeni's disease, von Recklinghausen's disease, tuberous sclerosis, xeroderma pigmentation, or retinoblastoma $[6,16,17]$.

The clinical, operative, and radiological records of the patients were reviewed, and data on the following variables were collected: clinical and treatment history before RIG diagnosis, clinical and treatment history after RIG diagnosis, date of operation for RIG, postoperative therapy for RIG, date of tumor recurrence of RIG, date of death or last hospital visit, extent of resection of RIG, and treatment after tumor recurrence of RIG. The extent of resection of the RIGs was determined based on the surgeon's operative notes and postoperative imaging studies and classified as follows: total, if $100 \%$ of the contrastenhanced lesion was resected; subtotal, if $95-99 \%$ of the lesion was resected; partial, if $<94 \%$ of the lesion was resected, or removed as a biopsy [18]. All patients were re-diagnosed by neuropathologists at our hospital according to the World Health Organization 2016 classification [19].

\section{Genetic analysis}


Tumor DNA was extracted from frozen tumor tissues in all cases using a DNeasy Blood \& Tissue Kit (Qiagen; Tokyo, Japan). The presence of hotspot mutations in the isocitrate dehydrogenase (IDH) 1 (R132) and IDH2 (R172) genes was assessed by pyrosequencing, as described previously [20]. Pyrosequencing assays were designed to detect all known mutations in these genes [20]. The two mutation hotspots in the telomerase reverse transcriptase (TERT) gene promoter were analyzed in all tumors using Sanger sequencing and/or pyrosequencing, as reported previously [21]. The mutation hotspots at codons 27 and 34 of the histone H3.3 (H3F3A) gene, and those at codon 600 of the B-Raf (BRAF) gene, were analyzed using Sanger sequencing and/or pyrosequencing [22]. The methylation status of the 0-6-methylguanine DNA methyltransferase (MGMT) promoter was analyzed using bisulfite modification of the tumor genomic DNA, followed by pyrosequencing, as previously described [21]. Methylation was considered positive when its mean level at the $16 \mathrm{CpG}$ sites was greater than $16 \%[18$, 21].

\section{Statistical analysis}

The latency period was defined as the interval between the date of diagnosis of the primary disease and that of RIG. Overall survival time (OS) was defined as the interval between the date of RIG surgery and that of death or the last follow-up, whichever occurred first. Progression-free survival time (PFS) was defined as the period between the date of RIG surgery and that of the detection of progression, death, or last follow-up. These times were calculated using the Kaplan-Meier method and compared using the logrank test. Statistical significance was set at $p<0.05$. Statistical tests were performed using JMP® ver. 15.1.0 software for Mac (SAS Institute Japan; Tokyo, Japan) and GraphPad Prism® ver. 9.0 (GraphPad Software; La Jolla, CA, USA).

\section{Results}

\section{Patient characteristics of primary disease}

We identified 11 patients who satisfied Cahan's criteria and had RIG $[6,16,17]$. The patient characteristics of the primary disease are summarized in Table 1. The median age of the 11 patients was 12 years (range: 1-39 years), and there was no sex predominance (male: 6 , female: 5). The primary diseases included germinomas $(n=2)$, acute lymphoblastic leukemias $(n=2)$, medulloblastomas $(n=3)$, diffuse astrocytoma with $I D H 2$ mutant $(n=1)$, pilocytic astrocytoma $(n=1)$, pituitary adenoma $(n=1)$, and a metastatic brain tumor from lung cancer $(n=1)$. All patients received cranial radiation (range: $12-101.5$ Gy). The median latency time between the primary disease and RIG was 17 years (range: 9-30 years) (Table 1).

\section{Patient characteristics and treatment of RIGs}

The characteristics of the patients with RIG are summarized in Table 2. The median age of the 11 patients was 34 years (range: 10-49 years). The RIGs included glioblastoma (GBM) with IDH1/2 wildtype $(n=7)$, GBM not otherwise specified $(n=2)$, anaplastic astrocytoma with $I D H 1 / 2$ wild-type $(n=1)$, and 
anaplastic astrocytoma not otherwise specified $(n=1)$. All patients underwent tumor removal or biopsy and were diagnosed based on histopathological examination. Two patients had multiple intraparenchymal lesions (Case 3 and Case 6), and one had right cerebellar and pontine lesions with cerebrospinal dissemination (Case 4).

For postoperative treatment, 5 patients received ReRT combined with chemotherapy, including ReRT/temozolomide (TMZ; n=2), ReRT/TMZ/bevacizumab (Bev; n=2), and ReRT/nimustine hydrochloride (ACNU; $n=1) ; 6$ patients were treated with chemotherapy alone, including TMZ $(n=4), \operatorname{ACNU}(n=1)$, and carboplatin and etoposide ( $n=1)$. The ReRT regimens were as follows: $40 \mathrm{~Gy}$ in 15 fractions, $40 \mathrm{~Gy}$ in 20 fractions, 50 Gy in 25 fractions, 60 Gy in 30 fractions, and 66 Gy in 33 fractions.

Ten patients had tumor recurrences, and 7 patients received further treatments for recurrent tumors. All the patients were treated with chemotherapy. Four patients received ReRT at the initial location at the time of recurrence. Two patients with supratentorial tumors had tumor recurrence in the cerebellum at a distance from the initial location and received stereotactic radiotherapy consisting of $42 \mathrm{~Gy}$ in 7 fractions (Table 2).

\section{Treatment outcomes of RIGs}

The median PFS and median survival time (MST) in patients with RIG were 11.3 months and 28.3 months, respectively (Figure 1A and 1B). The median PFS in patients initially treated with ReRT combined with chemotherapy $(n=5)$ was 17.0 months; this was longer than that of patients treated with chemotherapy alone (8.1 months, $n=6)$, although the difference was not significant $(p=0.45)$ (Figure 1C). The MST in patients initially treated with ReRT combined with chemotherapy $(n=5)$ and those receiving chemotherapy alone $(n=6)$ were 29.6 and 27.4 months, respectively, and were not significantly different $(p=0.27)$ (Figure 1D).

The tumor recurrence pattern of 9 patients after initial treatment for RIGs, excluding 1 patient who presented with cerebrospinal dissemination and 1 patient who did not have recurrence, were evaluated by radiological examinations. Among 4 patients who were treated initially with ReRT combined with chemotherapy, 2 had local recurrence (50\%), and 2 had distant recurrence (50\%); all 5 patients who were treated with chemotherapy alone had local recurrence $(100 \% ; p=0.046)$. None of the patients developed symptomatic radiation necrosis, which could be caused by a high cumulative radiation dose.

\section{Genetic alterations of RIGs}

The genetic alterations of 8 patients whose tumor samples were available for analysis are summarized in Table 3. There were no alterations in the IDH1/2 or TERT promoters in all 8 cases, and no BRAF or H3F3A mutations were found in the 6 cases for which data was available. Two tumors had MGMT promoters that were hypermethylated, whereas the other six had hypomethylated promoters.

\section{Case presentation 1}


A 4-year-old boy (Case 11) initially presented with headache, vomiting, and conscious disturbance, and underwent total removal of a right cerebellar tumor. The tumor was diagnosed as a medulloblastoma, and combined chemotherapy was performed with whole-brain radiation of $55.8 \mathrm{~Gy}$ in 31 fractions and whole-spine radiation of $23.4 \mathrm{~Gy}$ in 13 fractions. Twenty-four years after the treatment for medulloblastoma, the patient presented with dizziness; an MRI exam revealed a left cerebellar contrastenhanced tumor (Figure 2A, B). He underwent biopsy and was diagnosed as having GBM with IDH1/2 wild-type. He received ReRT at a dose of $40 \mathrm{~Gy}$ in 15 fractions combined with TMZ/Bev and maintenance $\mathrm{TMZ} / \mathrm{Bev}$ therapy. The tumor showed a complete response, and the patient did not develop tumor recurrence 9.8 months after the treatment for GBM with IDH1/2 wild-type (Figure 2C, D).

\section{Case presentation 2}

A 12-year-old female patient (Case 9) initially presented with a cataplectic attack; 3 years later, an MRI exam revealed a left frontal non-contrast-enhanced tumor (Figure 3A). She underwent subtotal resection and was diagnosed with diffuse astrocytoma. After the operation, she received radiation therapy at a dose of $60 \mathrm{~Gy}$ in 30 fractions and chemotherapy with ACNU. Seventeen years after treatment for the diffuse astrocytoma, she developed tumor recurrence just posterior to the primary tumor (Figure 3B) and underwent biopsy. The tumor was diagnosed as GBM with IDH1/2 wild-type (Figure 3C). We performed pyrosequencing analysis of the IDH1/2 gene in the primary tumor and found that the tumor had an IDH2 mutation (Figure 3D). Based on the difference in the IDH1/2 mutational states, we thought that the current GBM with IDH1/2 wild-type did not develop from the primary diffuse astrocytoma with IDH2 mutant; it was likely to be a de novo tumor that was related to the previous radiation therapy. Therefore, we diagnosed the GBM with IDH1/2 wild-type as RIG. The patient received ReRT at a dose of 60 Gy in 30 fractions combined with TMZ; however, the patient had a distant recurrence in the cerebellum 23.0 months after the treatment for GBM with $I D H 1 / 2$ wild-type (Figure 3E) and died 12.1 months thereafter.

\section{Discussion}

In this study, we observed a median latency time of 17 years, with a range of 9 to 30 years, suggesting the importance of a long follow-up period. Among the 11 patients with RIG, ReRT combined with chemotherapy was performed in 5 patients at the initial treatment of RIG and for 6 patients at the time of recurrence; the median PFS and MST were 11.3 months and 28.3 months, respectively. The local recurrences were observed less frequently in patients receiving ReRT combined with chemotherapy than in those receiving chemotherapy alone. We identified no genetic alterations in the IDH1/2 and TERT promoters or in the H3F3A, and BRAF genes; these are commonly mutated in spontaneous high-grade gliomas. In addition, we found that evaluation of the $I D H 1 / 2$ mutational status was helpful for establishing the diagnosis of RIG in cases whose IDH1/2 mutational states were different between the primary and secondary glioma.

The median latency time from the primary cancer treatment to the development of RIG was 17 years and varied from 9 to 30 years in our cohort. Previous studies reported that the median latency period was 911 years, and the incidence of RIG largely disappeared after 15-20 years [4, 5]. However, in our series, 4 
out of 11 patients (36.4\%) had a latency period of 20 years or more. Nakao et al. also reported that the latency period was more than 20 years in 4 patients [13]. These results indicate that pediatric patients with primary diseases that were treated successfully with radiation therapy have a risk of developing a secondary neoplasm more than 20 years after the initial treatment. From the clinical point of view, these patients should be carefully monitored much longer than we have expected; based on our results, we conclude that the follow-up time should be extended to 30 years.

One of the controversies in the treatment of RIG is whether ReRT has a therapeutic effect on tumors that were induced by previous radiation. In our study, 5 patients underwent ReRT during the initial treatment of RIG, and 6 patients had ReRT at the time of RIG recurrence. The median PFS and MST were 11.3 and 28.3 months, respectively, which were better than are those reported in previous studies $[5,6,23]$. In addition, patients initially receiving ReRT combined with chemotherapy tended to have a longer PFS than those receiving chemotherapy alone did, but MST was similar between the groups. The use of ReRT at the time of recurrence might prolong the OS in patients who have not undergone initial ReRT. Moreover, we found the local recurrences were observed less frequently in patients receiving ReRT combined with chemotherapy than in those receiving chemotherapy alone, suggesting a therapeutic effect on local control from ReRT. These observations are key findings, as they emphasize the importance of ReRT in the treatment of RIG, regardless of the timing. Paulino et al. reported that patients who underwent ReRT for RIG showed better survival rates than those who did not (13 vs. 8 months; $p=0.0009)$, suggesting that ReRT was efficacious in treating these tumors [5]. Yamanaka et al. reported that the MST of patients who received surgery, chemotherapy, and ReRT was 18 months; those who did not undergo chemotherapy had an MST of 9 months $(p=0.0006)$, suggesting that the combination of ReRT and chemotherapy is a potential treatment option for RIG [6]. Recent molecular analyses suggest that RIGs harbor a largely homogeneous genetic and epigenetic profile of sporadic pediatric receptor tyrosine kinase I in GBM, which might be particularly vulnerable to ionizing radiation $[14,15,24]$. While clinical and genetic evidence support that ReRT is a potential therapeutic option for the treatment of RIG, further studies using large cohorts are needed to confirm the efficacy of ReRT.

Another concern in the treatment of RIG is ReRT-related radiation necrosis. There was no symptomatic radiation necrosis observed in our patients. According to literature reviews, the risk of developing necrosis is less than $10 \%$ in the patients who underwent ReRT treatment for RIG [5]. The period between the first and second radiation sessions is usually more than 10 years, and most patients with RIG die within 3 years; therefore, late complications related to ReRT might not be clinically relevant. Two patients (Cases 10 and 11) in our cohort received Bev treatment combined with postoperative ReRT and TMZ, and they did not develop symptomatic radiation necrosis during the follow-up period. In Case 11, ReRT combined with $\mathrm{TMZ} /$ Bev after biopsy showed significant therapeutic efficacy without any sign of radiation necrosis (Figure 2). These cases suggest that the use of Bev combined with ReRT may be a promising therapeutic option for minimizing radiation-related risks with favorable therapeutic efficacy. It will be of interest to investigate the efficacy of combined ReRT and TMZ/Bev therapy in larger patient cohort. 
We investigated genetic alterations in 8 patients whose tumor samples were available. We found no alterations in the $I D H 1 / 2$ or TERT promoters or in the H3F3A or BRAFgenes; these are commonly altered in spontaneous high-grade gliomas. Two patients had a hypermethylated MGMT promoter; the other 6 patients had a hypomethylated MGMT promoter. These results are consistent with those of previous reports and confirm the genetic characteristics of RIG $[10-15,24]$. In Case 9, the difference in the IDH1/2 mutational status between the primary and secondary tumors was helpful for establishing the diagnosis of RIG. This case highlights the importance of determining the IDH1/2 mutational status in both primary and secondary gliomas. In addition, it is a reminder of the potential risk of developing RIG after radiation therapy for diffuse gliomas and the importance of long-term follow-up. Prior studies consistently report that RIGs hardly harbor IDH1/2 mutations [12-14]; therefore, identifying a wild-type IDH1/2 is diagnostically relevant. We recommend evaluating the IDH1/2 mutational status between the primary and secondary gliomas, especially in cases with recurrent glioma occurring a long time after a successful initial treatment.

Our study had certain limitations. First, this was a retrospective study, and the indications or dose/fraction regimens of ReRT were heterogeneous. They might have depended on the previous radiation field or dose/fraction regimen or period from the previous radiation; thus, heterogeneity was inevitable. Second, we did not investigate the genetic status in three patients because tissue samples were unavailable. Thus, further studies are needed to elucidate the genetic characteristics of RIG. Third, our cohort was too small to draw definitive conclusions. We acknowledge that the power of the survival analysis regarding the usefulness of ReRT, and the ReRT-related toxicity, was limited by the sample size; therefore, our results need to be confirmed in larger cohort studies.

\section{Conclusions}

RIG can occur more than 20 years after successful treatment of the primary disease using radiotherapy; thus, follow-up times should be extended to 30 years. ReRT combined with chemotherapy appears to be feasible and has favorable outcomes. ReRT combined with TMZ/Bev could be a promising therapeutic approach. Determining the IDH1/2 mutational status improves the diagnosis of RIG in recurrent glioma.

\section{Abbreviations}

RIG: radiation-induced glioma, ReRT: reirradiation, GBM: glioblastoma, TMZ: Temozolomide, Bev: Bevacizumab, ACNU: nimustine hydrochloride, IDH: isocitrate dehydrogenase, OS: overall survival, MST: median survival time, PFS: progression-free survival time, MGMT: 0-6-methylguanine DNA methyltransferase, TERT: telomerase reverse transcriptase, BRAF: B-Raf, H3F3A: histone H3.3

\section{Declarations}

\section{Ethics approval and consent to participants:}


All procedures performed in this study were in accordance with the ethical standards of the institutional review board and the 1964 Helsinki Declaration and its later amendments. This study was approved by the Institutional Review Board of the National Cancer Center (2004-066 or 2007-086). Written informed consent was obtained from all participants.

\section{Consent for publication:}

Not applicable.

\section{Availability of data and materials:}

The datasets used and/or analyzed during the current study are available from the corresponding author upon reasonable request.

\section{Competing interests:}

$\mathrm{HI}$ received grants from HekaBio, Elekta KK, and CICS, a consulting fee from HekaBio, and lecture fees from Varian, Itochu, and CICS. All authors report no disclosures relevant to the manuscript.

\section{Funding:}

None.

\section{Authors' contributions:}

$\mathrm{MO}$ and $\mathrm{YN}$ designed the study. MO, YM, MT, SY, YT, DK, MK, HI, and YN contributed to the patient management and treatment. AY and KS contributed to the diagnoses. MO, YM, MT, SY, YT, YM, KI, and YN contributed to sample collection, molecular analyses, data acquisition, and interpretation. MO conducted the statistical analyses. MO and YN wrote the manuscript. All the authors reviewed, edited, and approved the final manuscript.

\section{Acknowledgements:}

A part of this study was presented at the 19th International Symposium on Pediatric Neuro-Oncology meeting.

\section{References}

1. Carr CM, Benson JC, DeLone DR, Diehn FE, Kim DK, Merrell KW, et al. Intracranial long-term complications of radiation therapy: an image-based review. Neuroradiology. 2021;63:471-82.

2. Hijiya N, Hudson MM, Lensing S, Zacher M, Onciu M, Behm FG, et al. Cumulative incidence of secondary neoplasms as a first event after childhood acute lymphoblastic leukemia. JAMA. 2007;297:1207-15. 
3. Minniti G, Traish D, Ashley S, Gonsalves A, Brada M. Risk of second brain tumor after conservative surgery and radiotherapy for pituitary adenoma: update after an additional 10 years. J Clin Endocrinol Metab. 2005;90:800-4.

4. Neglia JP, Friedman DL, Yasui Y, Mertens AC, Hammond S, Stovall M, et al. Second malignant neoplasms in five-year survivors of childhood cancer: childhood cancer survivor study. J Natl Cancer Inst. 2001;93:618-29.

5. Paulino AC, Mai WY, Chintagumpala M, Taher A, Teh BS. Radiation-induced malignant gliomas: is there a role for reirradiation? Int J Radiat Oncol Biol Phys. 2008;71:1381-7.

6. Yamanaka R, Hayano A, Kanayama T. Radiation-induced gliomas: a comprehensive review and meta-analysis. Neurosurg Rev. 2018;41:719-31.

7. Neglia JP, Robison LL, Stovall M, Liu Y, Packer RJ, Hammond S, et al. New primary neoplasms of the central nervous system in survivors of childhood cancer: a report from the Childhood Cancer Survivor Study. J Natl Cancer Inst. 2006;98:1528-37.

8. Pettorini BL, Park YS, Caldarelli M, Massimi L, Tamburrini G, Di Rocco C. Radiation-induced brain tumours after central nervous system irradiation in childhood: a review. Childs Nerv Syst. 2008;24:793-805.

9. Hiraki T, Fukuoka K, Mori M, Arakawa Y, Matsushita Y, Hibiya Y, et al. Application of Genome-Wide DNA Methylation Analysis to Differentiate a Case of Radiation-Induced Glioblastoma From LateRelapsed Medulloblastoma. J Neuropathol Exp Neurol. 2021;80:552-57.

10. Izycka-Swieszewska E, Bien E, Stefanowicz J, Szurowska E, Szutowicz-Zielinska E, Koczkowska M, et al. Malignant Gliomas as Second Neoplasms in Pediatric Cancer Survivors: Neuropathological Study. Biomed Res Int. 2018;2018:4596812.

11. Kajitani T, Kanamori M, Saito R, Watanabe Y, Suzuki H, Watanabe M, et al. Three case reports of radiation-induced glioblastoma after complete remission of acute lymphoblastic leukemia. Brain Tumor Pathol. 2018;35:114-22.

12. Lopez GY, Van Ziffle J, Onodera C, Grenert JP, Yeh I, Bastian BC, et al. The genetic landscape of gliomas arising after therapeutic radiation. Acta Neuropathol. 2019;137:139-50.

13. Nakao T, Sasagawa Y, Nobusawa S, Takabatake Y, Sabit H, Kinoshita M, et al. Radiation-induced gliomas: a report of four cases and analysis of molecular biomarkers. Brain Tumor Pathol. 2017;34:149-54.

14. Deng MY, Sturm D, Pfaff E, Sill M, Stichel D, Balasubramanian GP, et al. Radiation-induced gliomas represent $\mathrm{H3}$-/IDH-wild type pediatric gliomas with recurrent PDGFRA amplification and loss of CDKN2A/B. Nat Commun. 2021;12:5530.

15. DeSisto J, Lucas JT, Jr., Xu K, Donson A, Lin T, Sanford B, et al. Comprehensive molecular characterization of pediatric radiation-induced high-grade glioma. Nat Commun. 2021;12:5531.

16. Cahan WG. Radiation-induced sarcoma--50 years later. Cancer. 1998;82:6-7.

17. Cahan WG, Woodard HQ, et al. Sarcoma arising in irradiated bone; report of 11 cases. Cancer. 1948;1:3-29. 
18. Ohno M, Miyakita $Y$, Takahashi M, Igaki $H$, Matsushita $Y$, Ichimura $K$, et al. Survival benefits of hypofractionated radiotherapy combined with temozolomide or temozolomide plus bevacizumab in elderly patients with glioblastoma aged $>/=75$ years. Radiat Oncol. 2019;14:200.

19. Louis DN, Ohgaki H, Wiestler OD, WK C. WHO classification of tumours of the central nervous system. Revise 4th edition. . Lyon: IARC Press. 2016.

20. Arita H, Narita Y, Matsushita Y, Fukushima S, Yoshida A, Takami H, et al. Development of a robust and sensitive pyrosequencing assay for the detection of IDH1/2 mutations in gliomas. Brain Tumor Pathol. 2015;32:22-30.

21. Arita H, Narita Y, Fukushima S, Tateishi K, Matsushita Y, Yoshida A, et al. Upregulating mutations in the TERT promoter commonly occur in adult malignant gliomas and are strongly associated with total 1p19q loss. Acta Neuropathol. 2013;126:267-76.

22. Arita H, Yamasaki K, Matsushita Y, Nakamura T, Shimokawa A, Takami H, et al. A combination of TERT promoter mutation and MGMT methylation status predicts clinically relevant subgroups of newly diagnosed glioblastomas. Acta Neuropathol Commun. 2016;4:79.

23. Salvati M, D'Elia A, Melone GA, Brogna C, Frati A, Raco A, et al. Radio-induced gliomas: 20-year experience and critical review of the pathology. J Neurooncol. 2008;89:169-77.

24. Whitehouse JP, Howlett M, Federico A, Kool M, Endersby R, Gottardo NG. Defining the molecular features of radiation-induced glioma: A systematic review and meta-analysis. Neurooncol Adv. 2021;3:vdab109.

\section{Tables}

Due to technical limitations, table 1 to 3 xls is only available as a download in the Supplemental Files section.

\section{Figures}




\section{Figure 1}

A

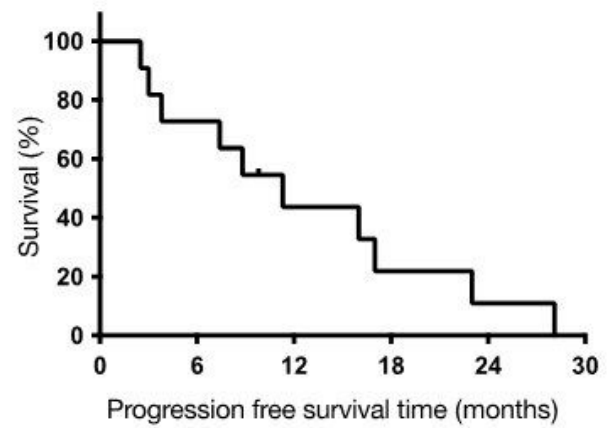

C

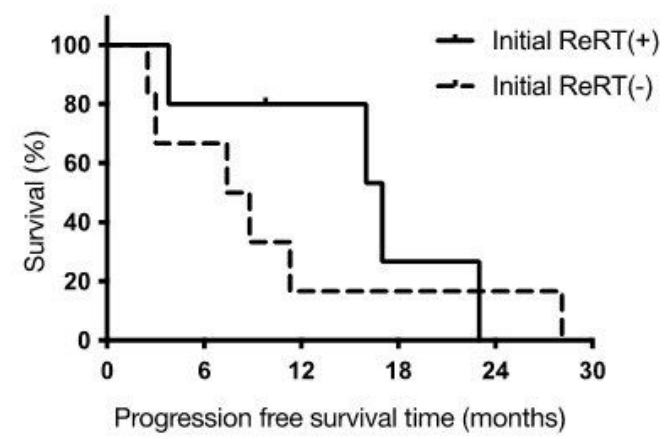

B

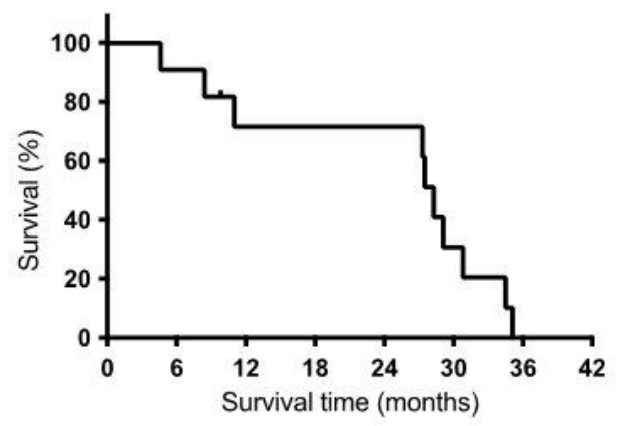

D

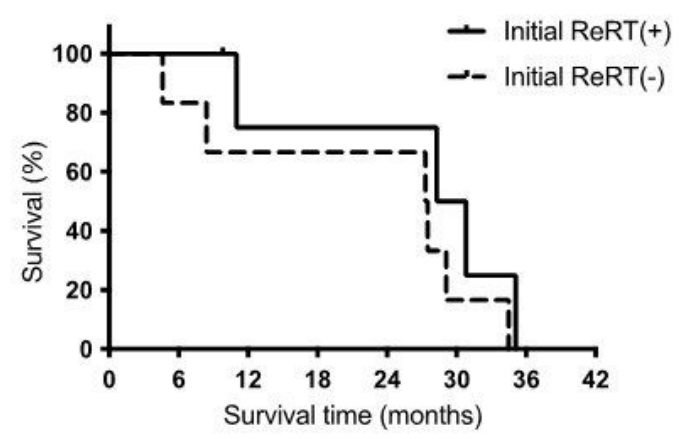

\section{Figure 1}

Kaplan-Meier curves of progression-free survival time (PFS) and overall survival time

(A) The median PFS was 11.3 months. (B) The median survival time was 28.3 months. (C) The median PFS in patients treated initially with reirradiation (ReRT) combined with chemotherapy $(n=5)$ was 17.0 months; this was longer than that of patients receiving chemotherapy alone (8.1 months, $n=6)$, although the difference was not significant $(p=0.45)$. (D) The median survival times of patients treated with ReRT combined with chemotherapy $(n=5)$ and those receiving chemotherapy alone $(n=6)$ were 29.6 and 27.4 months, respectively, and were not significantly different $(p=0.27)$ 


\section{Figure 2}
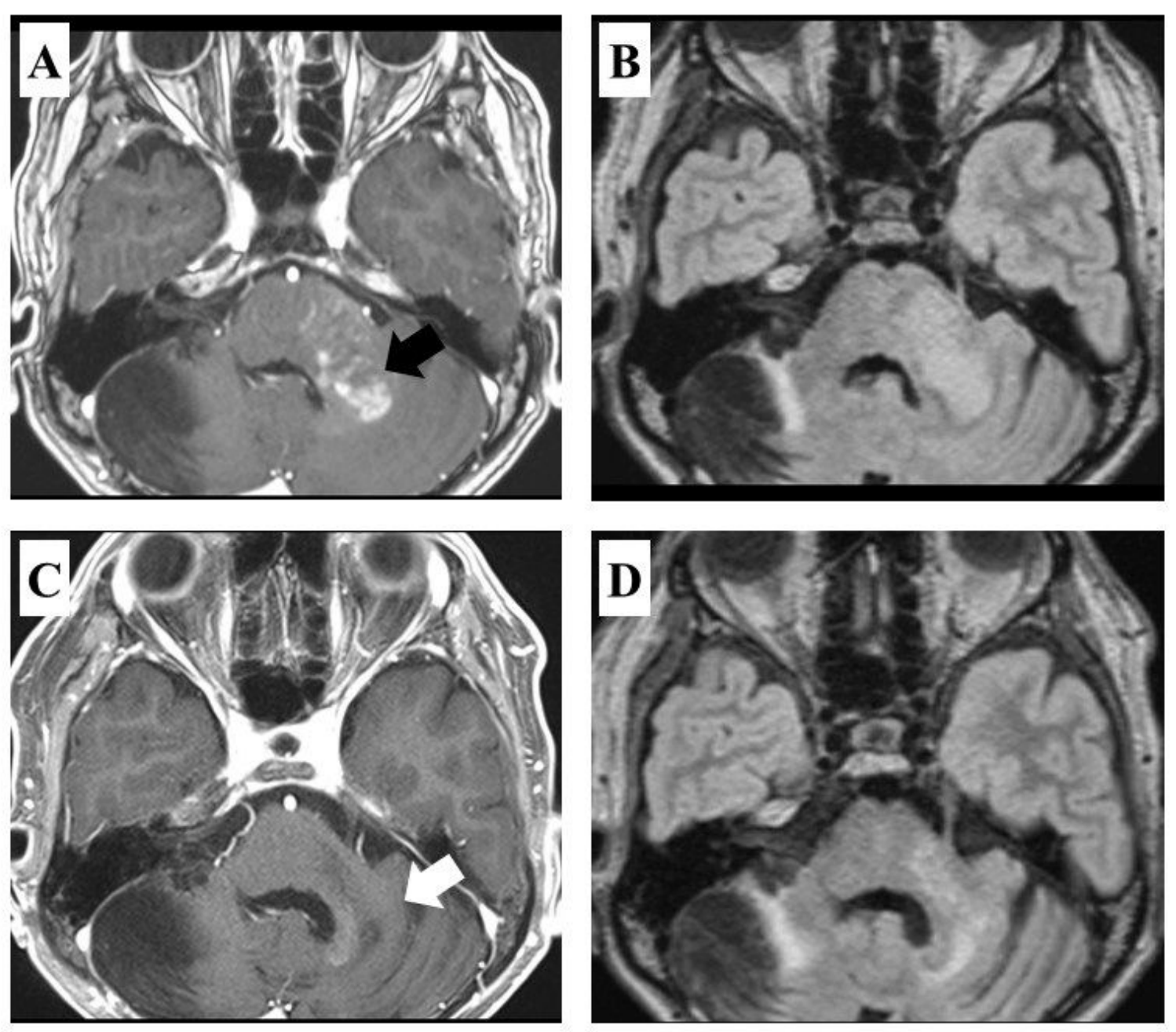

Figure 2

Representative patient treated with reirradiation, temozolomide, and bevacizumab (ReRT/TMZ/Bev) who showed a complete response

(A) Preoperative T1-weighted magnetic resonance image with gadolinium enhancement and (B) fluidattenuated inversion recovery (FLAIR) image showing an enhanced tumor in the left cerebellum (black arrow). (C) T1-weighted magnetic resonance image with gadolinium enhancement and (D) FLAIR image obtained 9.8 months after ReRT/TMZ/Bev treatment showing a complete response (white arrow). 


\section{Figure 3}
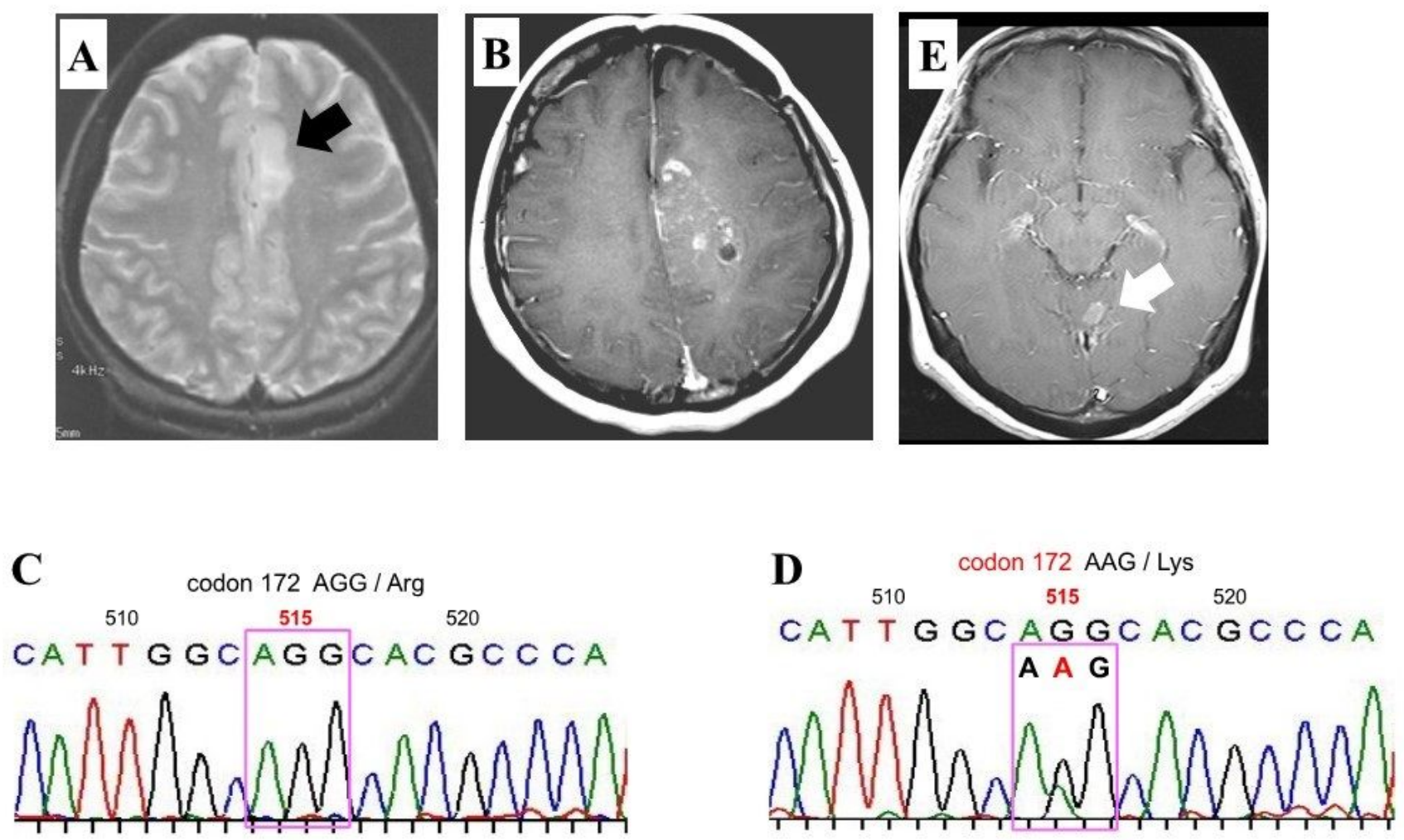

\section{Figure 3}

Representative patient treated with reirradiation and temozolomide (ReRT/TMZ) who showed a distant recurrence

(A) T2-weighted magnetic resonance image at the initial presentation showing a hyperintense lesion in the left medial frontal lobe (black arrow). (B) T1-weighted magnetic resonance image with gadolinium enhancement obtained 17 years after the primary tumor showing tumor recurrence just posterior to the primary tumor. (C) Sequencing analysis of a glioblastoma showing the homozygous $\mathrm{G}$ nucleotide at codon 515 of the IDH2 gene. (D) Sequencing analysis of a diffuse astrocytoma showing the heterozygous $\mathrm{G}$ and $\mathrm{A}$ nucleotides at codon 515 of the IDH2 alleles. (E) T1-weighted magnetic resonance images with gadolinium enhancement obtained 23.0 months after the secondary tumor diagnosis showing a distant lesion in the cerebellum (white arrow).

\section{Supplementary Files}

This is a list of supplementary files associated with this preprint. Click to download.

- 211225RIGTableROver1.0.xls 